\title{
Supramolecular balance: using cooperativity to amplify
}

\section{weak interactions}

Mihaela Roman,${ }^{\dagger}$ Caroline Cannizzo,${ }^{,}$Thomas Pinault,${ }^{,}$Benjamin Isare,${ }^{,}$Bruno Andrioletti,, Paul van der Schoot," and Laurent Bouteiller"

Université Claude Bernard-Lyon 1, ICBMS-UMR 5246, 43 Boulevard du 11 Novembre 1918, F-69622 Villeurbanne cedex, France

UPMC Univ Paris 06, UMR 7610, Chimie des Polymères, F-75005 Paris, France, and CNRS, UMR 7610, Chimie des Polymères, F-75005 Paris, France

"Group Theory of Polymers and Soft Matter, Technische Universiteit Eindhoven, P.O. Box 513, 5600

MB Eindhoven, The Netherlands, and Instituut voor Theoretische Fysica, Universiteit Utrecht, Leuvenlaan 4, 3584 CE Utrecht, The Netherlands

AUTHOR EMAIL ADDRESS laurent.bouteiller@upmc.fr; p.p.a.m.v.d.schoot@tue.nl

ABSTRACT Gathering precise knowledge on weak supramolecular interactions is difficult, yet is of utmost importance for numerous scientific fields, including catalysis, crystal engineering, ligand binding and protein folding. We report on a combined theoretical and experimental approach showing that it is possible to vastly improve the sensitivity of current methods to probe weak supramolecular interactions in solution. The concept consists of using a supramolecular platform involving a highly cooperative configurational transition, the perturbation of which (by the modification of the molecular building blocks) can be monitored in a temperature scanning experiment. We tested this concept with a particular bis-urea platform and our first results show that it is possible to detect the presence of 
interaction differences as low as $60 \mathrm{~J} / \mathrm{mol}$, which may be due to steric repulsion between vinyl and alkyl groups or may be the result of solvation effects.

\section{Introduction}

The precise knowledge of interactions that occur at the molecular level is of the utmost importance for numerous scientific fields. Indeed, manipulating non-covalent interactions is at the root of the whole field of supramolecular chemistry ${ }^{1}$ and has consequences in a wide range of scientific domains and practical applications. For instance, in crystal engineering, one tries to obtain crystal structures that are related in obvious ways to molecular structures and to achieve the ultimate goal of crystal structure prediction. However, crystal packing is not simply determined by a small number of strong interactions, but by the cooperation and competition of a large number of strong and weak interactions. ${ }^{2}$ In catalysis, the concept of manipulating weak attractive ligand-substrate interactions has been demonstrated. This has been implemented, for example, in the case of bifunctional catalysis and in olefin polymerization, where this approach has been shown to improve the control of the reactivity and the microstructure of the polymer chain. ${ }^{3}$ In the field of pharmaceuticals, a key step is to rationalize and optimize the interactions between a potential drug and a relevant receptor. In this context, the importance of interactions as weak as $1 \mathrm{~kJ} / \mathrm{mol}$ or less has been recognized. ${ }^{4}$ Finally, predicting the pathways of protein folding and quantifying the relative thermodynamic stability of intermediate and final states along these pathways, constitute two important challenges in modern chemistry.5

These examples illustrate that even weak interactions such as van der Waals interactions can collectively have a major influence on the structure and thus on the properties of a given assembly. It is therefore very important to be able to quantitatively measure weak molecular interactions. To this end, several clever synthetic systems have been devised to isolate and measure the energetic contribution of a particular interaction. For instance, a molecular torsion balance, based on a molecule with two restricted conformational states, allows one to probe weak intramolecular interactions through direct NMR measurement of the relative population of each state. ${ }^{6}$ Another possibility is to measure the association constant of a well-defined dimeric assembly. Incremental modification of the substituents can be used to probe a particular interaction, especially if a double-mutant cycle is performed to factor out unwanted secondary interactions.? These approaches have been very successful when it comes to the measurement 
of free energies for interactions such as hydrogen bonds, dipolar interactions or $\pi-\pi$ interactions. Unfortunately, an intrinsic limitation of these strategies is their limited sensitivity. In fact, the smallest uncertainty ever reported for these kind of studies is $0.12 \mathrm{~kJ} / \mathrm{mol}$, ${ }^{\text {tit }}$ and there is little hope to push this limit with the current methodology, even with an increase in NMR magnetic field. The limited sensitivity is due to the fact that the energetic information derives from the evaluation of concentrations (either of the two conformational states or of the intermolecular complexes), which are difficult to measure precisely. For example, biasing a molecular torsion balance with an interaction of $0.1 \mathrm{~kJ} / \mathrm{mol}$ means that the equilibrium population of the two conformations is shifted from a 50/50 to a 51/49 ratio. Measuring such a small shift by the integration of NMR signals is clearly difficult. Therefore, weaker interactions such as van der Waals interactions, isotope effects or chiral biases are currently out of reach by these approaches.

In biochemistry, interactions between DNA bases are commonly quantified by the measurement of the melting temperature of suitable DNA duplexes. ${ }^{8}$ Similarly, interactions between peptide residues are probed by comparing the denaturation temperature of related protein mutants, ${ }^{9}$ rather than the relative concentrations of folded and unfolded proteins, which would be much more difficult to quantify. In these cases, measuring a transition temperature is possible because transitions in these biochemical assemblies are cooperative, i.e., they assemble or disassemble within a narrow temperature range.

We propose to apply this successful approach to synthetic supramolecular systems, and the crucial idea for obtaining ultrahigh sensitivity to differences in interaction free energies is to use a cooperatively self-associating system as a platform. Incremental modification of the substituents will be used to probe a particular type of interaction, through variation of a suitable transition temperature of the platform that makes use of the competition between free monomers and two types of supramolecular polymer present in the solution. The achieved sensitivity of the measurement is due on the one hand to the high level of cooperativity of supramolecular polymerization of the chosen platform, and on the other to the competition between two types of assembly, i.e., the involvement of two equilibrium constants rather than one. The platform that we make use of is based on the self-organisation of bisureas in solution and enable us to distinguish the strength of van der Waals interactions between alkyl or alkene groups in an organic solvent. We put forward that any platform ${ }^{10}$ based on the same physical principles should be as effective as the one that we present here by way of proof of principle. 


\section{Results and Discussion}

Platform description. Bis-ureas consisting of a toluene central core bearing two urea moieties in 2 and 4 positions have been shown to self-assemble through hydrogen bonding into two distinct supramolecular structures of high molar masses." Remarkably, in low polarity solvents both structures have similar stabilities and are in fast dynamic exchange. ${ }^{12}$ Therefore, it is possible to switch the assembly from one structure to the other by changing the temperature. The high-temperature structure is a long filament with a single bis-urea in the cross-section (Figure 1a), ${ }^{13}$ while the low-temperature structure is a very long and rigid tube with three bis-ureas in the cross-section (Figure 1b)..$^{14}$ Both structures are characterized by a strongly cooperative growth, meaning that the formation of short oligomers is disfavored, compared to longer oligomers..$^{12,15}$ Because of this high level of cooperativity, the transition between tubes and filaments is very sharp and can conveniently be detected by an endothermic peak in a DSC experiment..$^{2}$ These features have been demonstrated for several bis-ureas bearing the same associating core, and seem to be common to this family of compounds. ${ }^{16}$ Moreover, because of the much tighter packing of the monomers in the case of the tube structure, intermolecular interactions within the tube can be expected to be significantly different from those within the filament. This system therefore constitutes an ideal platform to test our concept of a supramolecular balance, the theoretical principles of which we outline next. 
a)

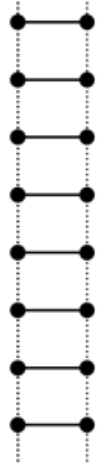

c)
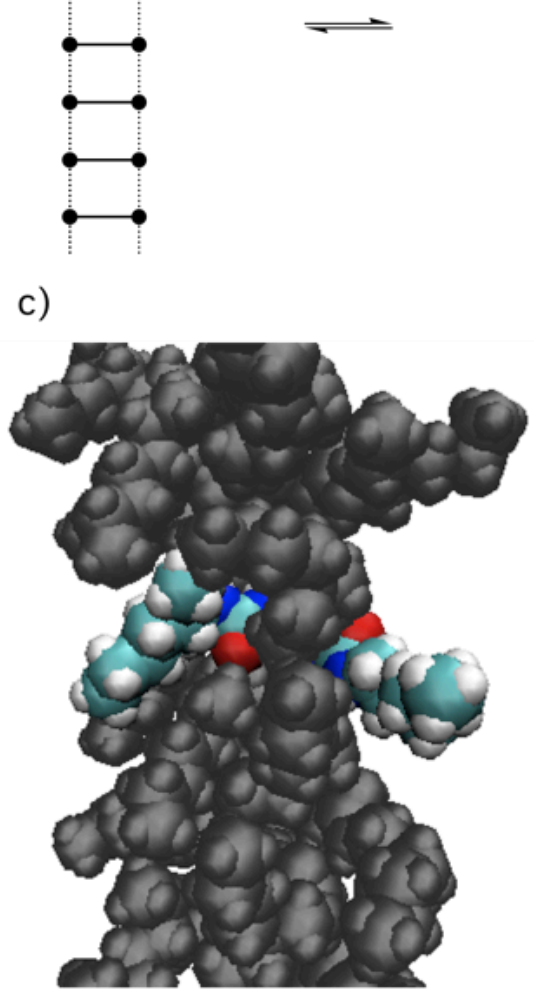
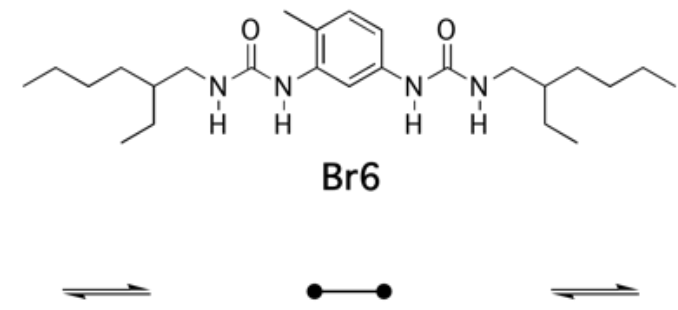

d)
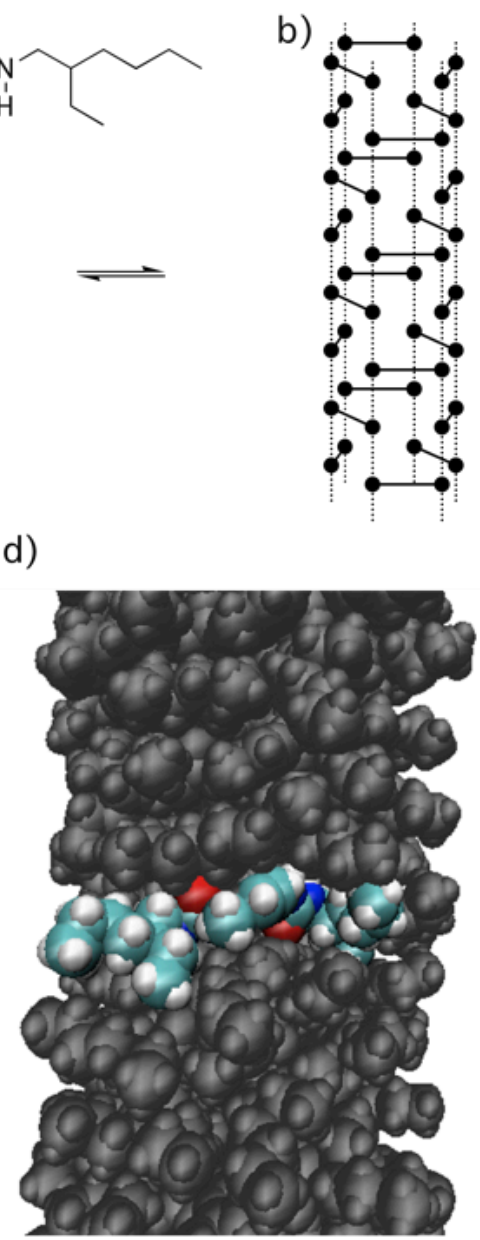

Figure 1. Schematic representations ( $a$ and b) and optimized geometries ${ }^{14 b}(c$ and d) for bis-urea $\mathbf{B r 6}$ in filament ( $\mathrm{a}$ and $\mathrm{c}$ ) or tube ( $\mathrm{b}$ and d) forms. In each assembly, a single bis-urea is highlighted with $\mathrm{O}$ in red, $\mathrm{N}$ in blue, $\mathrm{C}$ in light blue and $\mathrm{H}$ in white.

Theory. The key ingredient in the supramolecular balance is the ability of the monomer units to selfassemble in two competing types of supramolecular structure. At the (absolute) transition temperature $T=T_{0}^{\star \star}$ both types are equally stable and hence equally prevalent in the solution. Above (and below) this temperature one of them is more (less) stable than the other. As is shown explicitly in the Supporting Information, despite the presence of assemblies of all degrees of polymerization, the relative stability of these two distinct supramolecular structures turns out to be characterized by a single equilibrium constant $K_{0}=K_{0}(T)$ provided (i) the solution is dilute and (ii) their mean aggregation number is sufficiently large near the transition temperature $T_{0}^{*}$. This happens to be the case for sufficiently cooperative equilibrium polymerizations, even for conditions not too remote from the 
polymerization transition of either supramolecular structure. The (dimensionless) equilibrium constant $K_{0} \equiv \exp \left(-\Delta G_{0} / k_{B} T\right)$, with $k_{B}$ Boltzmann's constant, is a function of the free energy difference $\Delta G_{0}=\Delta G_{0}(T)$ of binding a single monomer in the two types of assembly. If $K_{0}<1$ or $K_{0}>1$ then one of the two states of aggregation predominates over the other. At the transition temperature we find that $K_{0}\left(T_{0}^{\star *}\right)=1$, again, provided the assemblies are in the mean of sufficiently large degree of polymerization. Although non-trivial and based (in essence) on the law of mass action, where we again refer to the SI for details, this result is actually quite intuitive. The reason is that at the transition from one to the other aggregate type, like in a true phase transition, the chemical potentials of the monomers in the two types of assembly must be equal. These chemical potentials are dominated by the respective binding free energies as the assemblies the monomers are part of have very little translational entropy. This implies that $\Delta G_{0}\left(T_{0}^{* *}\right)=0$, which is equivalent to the statement that $K_{0}\left(T_{0}^{\star *}\right)=1$. Strictly speaking, $\Delta G_{0}\left(T_{0}^{\star *}\right)$ is not quite zero but very small on the scale of the thermal energy $k_{B} T_{0}^{*}$ due to finite-size effects.

The temperature dependence of this equilibrium constant near its transition temperature is given by

$$
K_{0}(T)=K_{0}\left(T_{0}^{\star \star}\right) e^{\frac{\Delta h_{0}}{k_{B} T_{0}^{\star \star 2}}\left(T-T_{0}^{\star \star}\right)}=e^{\frac{\Delta h_{0}}{k_{B} T_{0}^{\star \star 2}}\left(T-T_{0}^{\star \star}\right)}
$$

where $\Delta h_{0} \equiv \Delta h_{0}\left(T_{0}^{* *}\right)$ is the enthalpy difference of a monomer bound to the two types of supramolecular structure, measured at the transition temperature. Eq (1) follows from a Taylor expansion and is valid for temperatures $\left|T-T_{0}^{* *}\right|<<T_{0}^{* *}$ and presumes the solution to be incompressible. See the SI. If we now consider a similar compound that can self-assemble into the same two competing supramolecular structures, then their relative stability can be expressed in a different equilibrium constant that we denote $K_{1}$. For this association constant we can write

$$
K_{1}(T)=K_{1}\left(T_{0}^{\star \star}\right) e^{\frac{\Delta h_{1}}{k_{B} T_{0}^{\star * 2}}\left(T-T_{0}^{\star \star}\right)}
$$


for temperatures near the transition temperature of the other component, so $\left|T-T_{0}^{\star *}\right| \ll<T_{0}^{\star *}$, with $\Delta h_{1} \equiv \Delta h_{1}\left(T_{0}^{* *}\right)$ again an enthalpy difference measured at the temperature $T_{0}^{\star *}$. Here, we have taken the transition of component 0 as reference point. As we have seen, at the respective transition temperatures of the supramolecular structures for the two compounds, $T_{0}^{* *}$ and $T_{1}^{* *}$, the equilibrium constants are, to leading order in the reciprocal aggregation numbers and at equal overall concentration of monomers, equal. Hence, equating eqs (1) and (2) gives

$$
K_{0}\left(T_{0}^{* *}\right)=K_{1}\left(T_{1}^{* *}\right)=K_{1}\left(T_{0}^{* *}\right) e^{\frac{\Delta h_{1}}{k_{B} T_{0}^{* \star 2}\left(T_{1}^{* \star}-T_{0}^{* \star}\right)}}
$$

and therefore

$$
\frac{\Delta h_{1}}{k_{B} T_{0}^{* * 2}}\left(T_{1}^{* *}-T_{0}^{* *}\right)=\ln K_{0}\left(T_{0}^{* *}\right)-\ln K_{1}\left(T_{0}^{* *}\right)=-\frac{\Delta G_{0}\left(T_{0}^{* *}\right)-\Delta G_{1}\left(T_{0}^{* *}\right)}{k_{B} T_{0}^{* *}}
$$

which leads to the central result of our analysis,

$$
\Delta \Delta G \equiv \Delta G_{1}\left(T_{0}^{* *}\right)-\Delta G_{0}\left(T_{0}^{* *}\right)=\Delta h_{1} \frac{T_{1}^{* *}-T_{0}^{* *}}{T_{0}^{* *}}
$$

According to eq (5), a structural change from compound $\mathbf{0}$ to compound $\mathbf{1}$ can be associated to a free energy difference that is the product of the transition enthalpy and the relative change in transition temperature, both of which can be measured as already indicated, e.g., in a DSC experiment. Because the temperature is in Kelvin units, a free energy change $\Delta \Delta G$ two orders of magnitudes lower than the transition enthalpy $\Delta h$ can easily be detected. Therefore, a very small free energy change can be derived from a precise transition temperature measurement on a platform having a suitably low (but measurable) transition enthalpy.

Interactions between alkyl chains. As a first test of our concept of the supramolecular balance, we decided to investigate the quantitative influence of the length of the alkyl substituents of the bis-urea monomers, which should inform us on the strength of van der Waals interactions between alkyl chains. Thus, a range of monomers was synthesized (DL2 to DL18, Chart 1) by reaction of 2,4toluenediisocyanate on $n$-alkylamines of various lengths. Unfortunately, none of these bis-ureas with linear alkyl substituents are soluble in non-polar solvents (such as cyclohexane, toluene or chloroform), 
so that the relative stability of the filament and tube forms cannot be probed. To improve solubility, branching was introduced either in one or in both substituents. In the former case, bis-urea monomers L1 to L16 bear a 2-ethylhexyl group on one side and a linear alkyl of given length on the other side. The dissymmetry in the monomers is derived from 2-amino-4-nitrotoluene (Scheme 1). In the latter case, bis-urea monomers Br5 to Br8 bear a 2-ethylalkyl group of given length on both sides of the molecule. In this case, the branched alkylamines were not commercially available and were synthesized by alkylation and reduction of butyronitrile (Scheme 2). Satisfyingly, all branched bis-ureas synthesized are soluble in toluene.

First of all, the supramolecular structures formed by these compounds in toluene were characterized by the combination of Small Angle Neutron Scattering (SANS) and FTIR spectroscopy (see Figures S1 and S2, and Table S1 in Supporting Information). All the bis-ureas studied were found to self-assemble into a quasi one-dimensional structure containing 3 molecules in the cross-section (and previously identified as the tube form) at low temperatures, and into filaments containing a single molecule in the cross-section at high temperatures. As expected, the minor changes introduced in the alkyl side-chains do not change the structure of the assemblies: the platform thus appears to be sufficiently robust. The relative stability of the two supramolecular structures was then probed by high-sensitivity DSC experiments. All solutions yield a narrow and reversible endothermal peak characteristic for the transition between tube and filament (see Figure S3 and Table S1 in Supporting Information). Remarkably, the minor changes introduced in the alkyl side-chains have a very strong effect on the value of the transition temperature, which spans the range 27 to $50^{\circ} \mathrm{C}$ (Figure $2 \mathrm{a}$ ). The transition temperatures and enthalpies measured by DSC can be converted into free energy differences, according to equation (5) (Figure 2b). ${ }^{18}$ The first striking result is that both series of compounds display a nonmonotonic variation of the free energy as a function of the alkyl chain length. Moreover, in both cases, the maximum of stability of the tube form is reached when the longest branch of the variable alkyl chain is composed of 6 carbon atoms. This indicates that two competing effects are involved when the length of the alkyl chains is altered, and that they cancel each other when the chains are 6 carbon atoms long. In principle, an increase of the tube to filament transition temperature can either be due to stabilizing interactions in the tube form or to destabilizing interactions in the filament form. ${ }^{19}$ However, in the filament form, a large distance between bis-ureas is imposed by the hydrogen-bonded core (4.6 $⿱$ ) ), so 
that it is reasonable to assume that alkyl chains interact less in the filament form than in the more compact tube form (Figure 1). Therefore, in the first approximation, we plausibly presume the filament to be unaffected by the alkyl chain length,,$^{21}$ and we attempt to correlate the stability of the tube to filament transition to interactions occurring in the tube form. Accordingly, the data of Figure 2 can be interpreted by the predominance of attractive van der Waals interactions among alkyl chains, if they are less than 6 carbon atoms long; and by that of steric repulsions among alkyl chains, if they are more than 6 carbon atoms long. Indeed, steric repulsion leads to reduced numbers of conformations and hence reduced conformational entropy, an effect that becomes stronger with increasing alkyl chain length. Furthermore, from the first part of the curve, it can be estimated that each additional methylene group contributes $70 \mathrm{~J} / \mathrm{mol}$ to the stabilization of the tube form. This value may seem surprisingly small compared to known values of van der Waals interaction, derived for instance from the Gibbs free energy of micellization of surfactants $(\Delta \mathrm{G}=3.5 \mathrm{~kJ} / \mathrm{mol}$ per methylene $) .^{22}$ However, one has to realize that the reference state is not the same. In the present case, we measure the free energy gain for a methylene group in the tube structure compared to the filament structure. Thus, in a first approximation, we measure the free energy gain for a methylene group interacting with alkyl groups compared to the situation where it is solvated by toluene (instead of being solvated by water, as is the case in micellization). 
Chart 1. Structure of the monomers.<smiles>Cc1ccc(NC(=O)[NH+]2CC3CCC2C3)cc1NC(=O)N1CCCC1</smiles>

$$
\begin{array}{ll}
\text { DL2 }: q=2 & \text { DL12: }: q=12 \\
\text { DL5 }: q=5 & \text { DL18 }: q=18 \\
\text { DL8 }: q=8 &
\end{array}
$$<smiles>CCC(CNC(=O)Nc1ccc(C)c(NC(=O)NNCC(CC)C2(CC)CCC2(CC)CC)c1)C(C)(CC)CC</smiles>

$$
\begin{array}{ll}
\text { Br5 }: n=5 & \text { Br7 }: n=7 \\
\operatorname{Br} 6: n=6 & \text { Br8 }: n=8
\end{array}
$$<smiles>CCCCC(CC)CNC(=O)Nc1cc(NC(=O)[NH+]2CC3CC[In]32)ccc1C</smiles>

$$
\begin{array}{ll}
\text { L1 }: m=1 & \text { L6: } m=6 \\
\text { L2 }: m=2 & \text { L8: } m=8 \\
\text { L3 }: m=3 & \text { L12:m=12 } \\
\text { L4 }: m=4 & \text { L16: } m=16
\end{array}
$$<smiles>C=CCCC(CC)CNNC(=O)Nc1cc(NC(=O)NCC(CC)CCC=C)ccc1C</smiles><smiles>C=CCCC(CC)CNC(=O)Nc1ccc(C)c(NC(=O)NNCC(CC)CCCC)c1</smiles> 
Scheme 1. Synthesis of monomers L1 to L16.

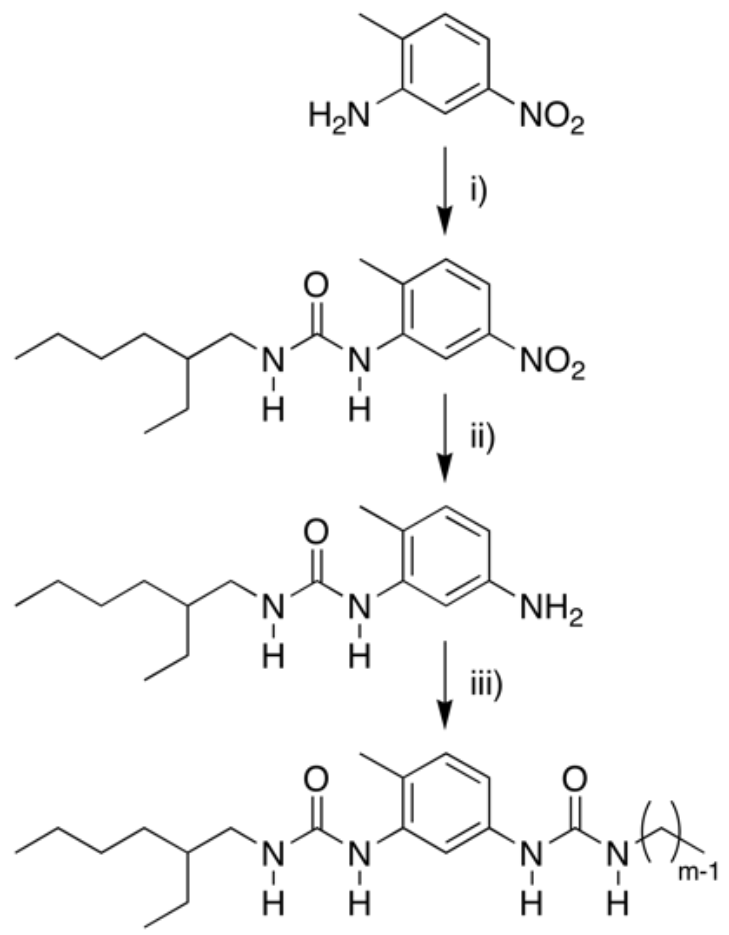

(i) 2-ethylhexylisocyanate, $\mathrm{CH}_{2} \mathrm{Cl}_{2}, \mathrm{RT}, 56 \%$; (ii) cyclohexene, $\mathrm{Pd} / \mathrm{C}$, isopropanol, 85\%; (iii) $\mathrm{C}_{\mathrm{m}} \mathrm{H}_{2 m+1} \mathrm{NCO}$ or $\mathrm{C}_{\mathrm{m}} \mathrm{H}_{2 m+1} \mathrm{NH}_{2}$, triphosgene, diisopropylethylamine, THF, RT, 11-64\%.

Scheme 2. Synthesis of monomers Br5 to Br8. ${ }^{a}$
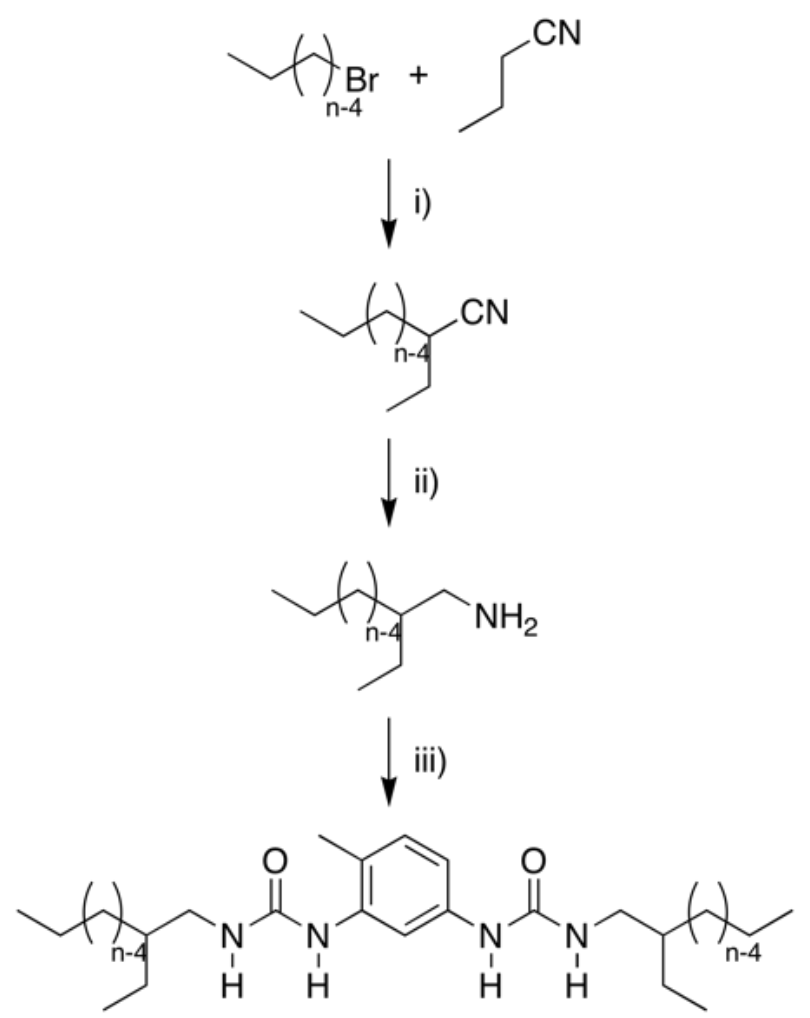

(i) $\mathrm{LDA}-78^{\circ} \mathrm{C}$ then $\mathrm{RT}, 41-88 \%$; (ii) $\mathrm{LiAlH}_{4}, \mathrm{Et}_{2} \mathrm{O}, 0^{\circ} \mathrm{C}$ then reflux, 66-85\%; (iii) toluene 2,4diisocyanate, $\mathrm{CH}_{2} \mathrm{Cl}_{2}, \mathrm{RT}, 41-93 \%$. 

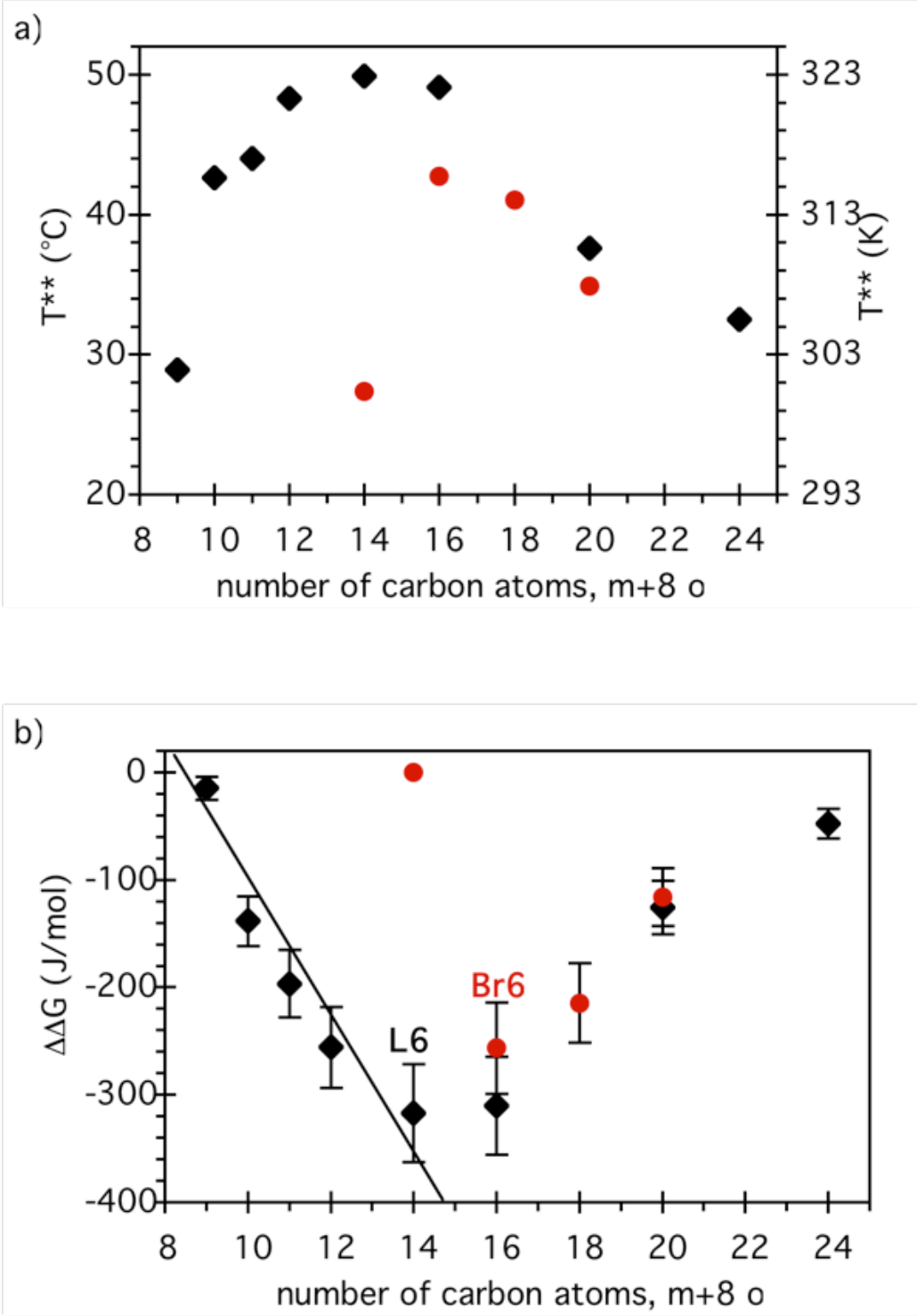

Figure 2. (a) Transition temperature for bis-ureas Br5 to Br8 (red) and bis-urea L1 to L16 (black), measured in toluene $(10 \mathrm{mM})$. (b) Relative free energy of the tube to filament transition, versus alkyl chain length (the reference is $\mathbf{B r 5}$ and the reference temperature is $27.4^{\circ} \mathrm{C}$ ).

Interactions involving alkene chains. Aromatic groups are well-known to interact by pi-stacking or weak hydrogen bonding. However, much less is known about interactions involving vinyl groups, 
certainly because of their weak intensity. Therefore, bis-urea DV6 (Chart 1) was synthesized to probe these interactions. The vinyl groups were introduced in terminal position of the alkyl substituents, because it was anticipated that at these positions, they would not induce any conformational bias in the monomer. DSC experiments (Figure 3 and S3) show that the tube to filament transition occurs at $31.5^{\circ} \mathrm{C}$, as compared to $42.7^{\circ} \mathrm{C}$ for bis-urea Br6. This 11 degree difference is much larger than the experimental error and unambiguously shows that replacing the terminal ethyl groups by terminal vinyl groups destabilizes the bis-ureas in the tube structure (or stabilizes the bis-ureas in the filament structure) by $110 \pm 15 \mathrm{~J} / \mathrm{mol}$. Not surprisingly, this value is low, however it is not negligible, and a better knowledge of this interaction could have an impact, for instance, on the design of polymerization catalysts. ${ }^{3}$

Our supramolecular balance is clearly of unprecedented sensitivity, but at the same time unfortunately hampered by our poor knowledge of the relative positions of the bis-ureas in the tube assembly. At this point, no crystal of the tube structure has been obtained, and the very broad NMR spectra of bis-ureas in toluene preclude NOE experiments. This is in fact the exactly symmetrical situation compared to crystallographic data analysis, which yields an unambiguous position for the atoms, but no direct information about the energetics of a particular interaction. In the case of crystallography, a careful statistical analysis of a large number of crystal structures makes it possible to isolate the energetic contribution of a particular interaction from the pervading crystal packing forces. In the present case, the build-up of a large data set on related compounds should make it possible to deduce also precise geometrical information.

Let us first assume that no differential solvation effect is present between the tube and filament forms. Then, as in the previous section, we can assume that the decrease of the tube to filament transition is due to destabilizing interactions in the tube form, rather than stabilizing interactions in the filament form. It is clear from the molecular simulations of the tube structure (Figure 1), ${ }^{14 \mathrm{~b} \cdot \mathrm{d}}$ that the vinyl groups can only come into contact with other vinyl groups or with alkyl groups. To discriminate whether the $110 \mathrm{~J} / \mathrm{mol}$ bias is due to vinyl/vinyl interactions or to vinyl/alkyl interactions, it is possible to design a suitable blank experiment. For instance, "diluting" the vinyl groups in the tube structure should lead to a reduction of both vinyl/vinyl and vinyl/alkyl contacts, but not in the same proportion. Accordingly, bisurea V6, with a single vinyl group (Chart 1), was synthesized and characterized. Because of the lack of 
symmetry of bis-urea V6, two orientations are possible for each monomer in the tube: the vinyl group can either be on the left or on the right. However, given the low value of the interaction considered, we can safely assume that the statistics are not biased by the interaction, so that the orientation of the monomer in the tube is random. In this case, the number of vinyl/vinyl contacts for V6 should be a quarter of the value for DV6. In contrast, in the tube structure, the vinyl group of V6 is always in the vicinity of methylene or methyl groups from its neighbors; therefore, the number of vinyl/alkyl contacts for V6 should be half the value for DV6. ${ }^{23}$ The DSC experiment (Figure 3 and S3) shows that the tube to filament transition occurs at $38.0^{\circ} \mathrm{C}$ for bis-urea $\mathbf{V 6}$, as compared to $31.5^{\circ} \mathrm{C}$ and $42.7^{\circ} \mathrm{C}$ for bis-ureas DV6 and Br6, respectively. This median value means that replacing a single terminal ethyl group per bis-urea by a terminal vinyl group destabilizes the tube structure by $60 \pm 10 \mathrm{~J} / \mathrm{mol}$, i.e. about half the value for DV6. It can therefore be concluded that the destabilization is consistent with repulsive interactions between vinyl and methyl or methylene groups. Of course, it is difficult to attribute more precisely the nature of this interaction but it may plausibly be related to an unfavorable packing of the vinyl and alkyl groups, by analogy to the melting point depression of unsaturated fatty acids.

Let us now examine possible solvation effects. The filament form is less compact than the tube form, therefore it is likely that the vinyl groups in the filament form are more exposed to the solvent than in the tube form. If this is the case, then the decrease in the tube to filament transition can be due to favorable interactions between the vinyl groups and the solvent (toluene). The linearity of the effect on going from Br6 to V6 and to DV6 (Figure 3b) is compatible with this interpretation.

We have thus identified two plausible interpretations: steric repulsion between vinyl and alkyl groups in the tube form or favorable solvation of the vinyl groups by toluene in the filament form. Reproducing the experiment in a range of solvents may help to discriminate between the two interpretations. 


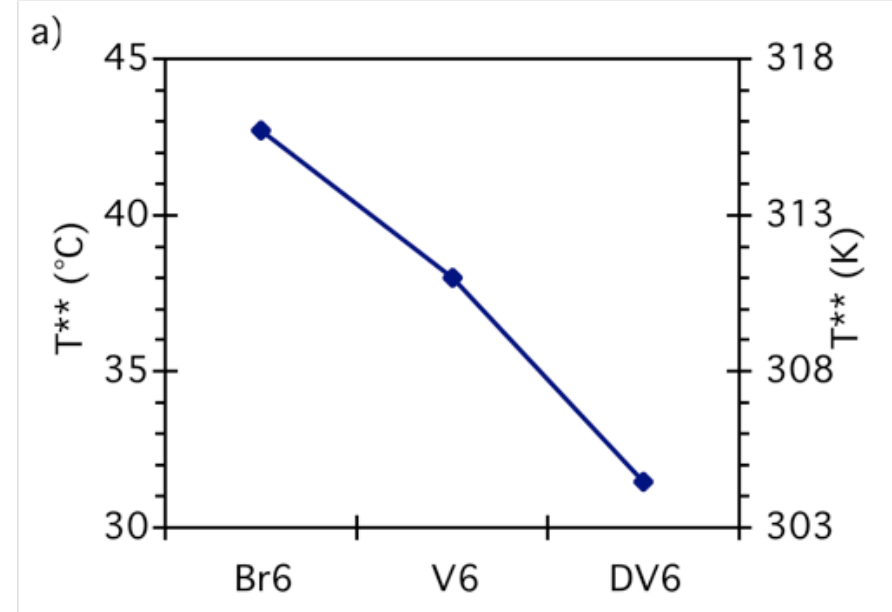

b)

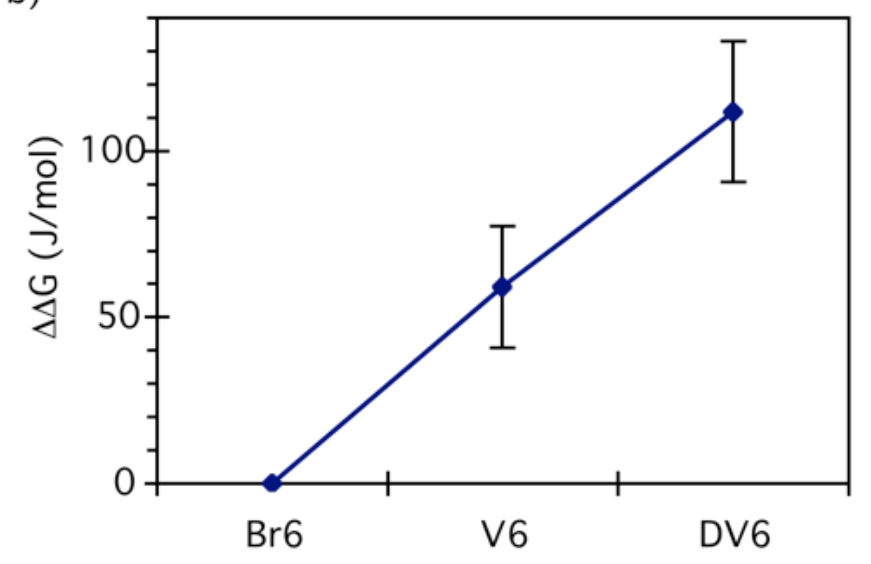

Figure 3. (a) Transition temperature measured in toluene $(10 \mathrm{mM})$. (b) Relative free energy of the tube to filament transition (the reference is Br6 and the reference temperature is $42.7^{\circ} \mathrm{C}$ ).

\section{Conclusion}

In conclusion, we report a theoretical analysis showing that it is possible to vastly improve the sensitivity of current methods to measure weak supramolecular interactions in solution. The concept consists in using a supramolecular platform, which presents a cooperative transition, so that a perturbation of this transition can be monitored by a temperature scanning experiment. This concept has been tested with a particular bis-urea platform. Our first results show that it is possible to detect the presence of interactions as low as $60 \mathrm{~J} / \mathrm{mol}$, which may occur between vinyl and alkyl groups, or be the 
result of solvation effects. At present, the weakness of our proposed supramolecular balance is the poor knowledge of the relative position of the interacting groups, but it is our opinion that the build up of a large data set on related compounds will make it possible to deduce also precise geometrical information. Moreover, the exceptional sensitivity of this platform can also be used to probe weak interactions between solvent molecules in solvent mixtures..$^{24}$

ACKNOWLEDGMENT Financial support from the French ANR program (ANR-06-BLAN-0159 zeoliplastic) is acknowledged. Guillaume Zagury is thanked for his contribution to the synthesis of bisureas. We thank François Boué (LLB, Saclay) for assistance with SANS experiments.

SUPPORTING INFORMATION AVAILABLE Theory of competing supramolecular assembly; Experimental Section: synthesis and characterization of bis-ureas; SANS, FTIR, DSC and ITC data. This information is available free of charge via the Internet at http://pubs.acs.org/.

\section{REFERENCES}

(1) (a) Lehn, J.-M. Proc. Natl. Acad. Sci. USA 2002, 99, 4763-4768. (b) Ling, X. Y.; Reinhoudt, D. N.; Huskens, J. Pure Appl. Chem. 2009, 81, 2225-2233. (c) Sijbesma, R. P.; Beijer, F. H.; Brunsveld, L.; Folmer, B. J. B.; Hirschberg, J. H. K. K.; Lange, R. F. M.; Lowe, J. K. L.; Meijer, E. W. Science 1997, 278, 1601-1604.

(2) Desiraju, G. R.; Steiner, T. The weak hydrogen bond, Oxford University Press, 1999.

(3) (a) Ikariya, T.; Blacker, A. J. Acc. Chem. Res. 2007, 40, 1300-. (b) Chan, M. C. W. Chem. Asian $J .2008,3,18-27$.

(4) Müller, K.; Faeh, C.; Diederich, F. Science 2007, 317, 1881-1886.

(5) (a) Baltzer, L.; Nilsson, H.; Nilsson, J. Chem. Rev. 2001, 101, 3153-3163. (b) Shakhnovich, E. Chem. Rev. 2006, 106, 1559-1588.

(6) (a) Paliwal, S.; Geib, S.; Wilcox, C. S. J. Am. Chem. Soc. 1994, 116, 4497-4498. (b) Nakamura, K.; Houk, K. N. Org Lett. 1999, 1, 2049-2051. (c) Cockroft, S. L.; Hunter, C. A. Chem. Commun. 2006, 
3806-3808. (d) Gung, B. W.; Xue, X.; Zou, Y. J. Org. Chem. 2007, 72, 2469-2475. (e) Bhayana, B.; Wilcox, C. S. Angew. Chem. Int. Ed. 2007, 46, 6833-6836. (f) Fischer, F. R.; Schweizer, W. B.; Diederich, F. Angew. Chem. Int. Ed. 2007, 46, 8270-8273. (g) Fischer, F. R.; Schweizer, W. B.; Diederich, F. Chem. Commun. 2008, 4031-4033. (h) Fischer, F. R.; Wood, P. A.; Allen, F. H.; Diederich, F. Proc. Natl. Acad. Sci. USA 2008, 105, 17290-17294. (i) Carroll, W. R.; Pellechia, P.; Shimizu, K. D. Org Lett. 2008, 10, 3547-3550. (j) Cornago, P.; Claramunt, R. M.; Bouissane, L.; Elguero, J. Tetrahedron 2008, 64, 3667-3673. (k) Aliev, A. E.; Moïse, J.; Motherwell, W. B. Phys. Chem. Chem. Phys. 2009, 11, 97-100. (1) Cockroft, S. L.; Hunter, C. A. Chem. Commun. 2009, 39613963. (m) For a review, see: Mati, I. K.; Cockroft, S. L. Chem. Soc. Rev. 2010, Advance Article, DOI: $10.1039 / \mathrm{b} 822665 \mathrm{~m}$.

(7) (a) Adams, H.; Carver, F. J.; Hunter, C. A.; Morales, J. C.; Seward, E. M. Angew. Chem. Int. Ed. 1996, 35, 1542-1544. (b) For a review, see: Cockroft, S. L.; Hunter, C. A. Chem. Soc. Rev. 2007, 36, $172-188$.

(8) (a) Petersheim, M.; Turner, D. H. Biochemistry 1983, 22, 256-263. (b) Guckian, K. M.; Schweitzer, B. A.; Ren, R. X.-F.; Sheils, C. J.; Paris, P. L.; Tahmassebi, D. C.; Kool, E. T. J. Am. Chem. Soc. 1996, 118, 8182-8183.

(9) (a) Fernandez, A. M.; Villegas, V.; Martinez, J. C.; van Nuland, N. A. J.; Conejero-Lara, F.; Aviles, F. X.; Serrano, L.; Filimonov, V. V.; Mateo, P. L. Eur. J. Biochem. 2000, 267, 5891-5899. (b) Vassall, K. A.; Stathopulos, P. B.; Rumfeldt, J. A. O.; Lepock, J. R.; Meiering, E. M. Biochemistry 2006, 45, 7366-7379.

(10) For example: (a) Brunsveld, L.; Zhang, H.; Glasbeek, M.; Vekemans, J. A. J. M.; Meijer, E. W. J. Am. Chem. Soc. 2000, 122, 6175-6182. (b) van der Schoot, P.; Michels, M. A. J.; Brunsveld, L.; Sijbesma, R. P.; Ramzi, A. Langmuir 2000, 16, 10076-10083.

(11) Bouteiller, L.; Colombani, O.; Lortie, F.; Terech, P. J. Am. Chem. Soc. 2005, 127, 8893-8898.

(12) Bellot, M.; Bouteiller, L. Langmuir 2008, 24, 14176-14182. 
(13) (a) Vonau, F.; Suhr, D.; Aubel, D.; Bouteiller, L.; Reiter, G.; Simon, L. Phys. Rev. Lett. 2005, 94, 066103. (b) Vonau, F.; Aubel, D.; Bouteiller, L.; Reiter, G.; Simon, L. Phys. Rev. Lett. 2007, 99, 086103. (c) Vonau, F.; Linares, M.; Isare, B.; Aubel, D.; Habar, M.; Bouteiller, L.; Reiter, G.; Geskin, V.; Zerbetto, F.; Lazzaroni, R.; Simon, L. J. Phys. Chem. C 2009, 113, 4955-4959.

(14) (a) Pinault, T.; Isare, B.; Bouteiller, L. Chem. Phys. Chem. 2006, 7, 816-819. (b) Shikata, T.; Nishida, T.; Lazzaroni, R.; Bouteiller, L. J. Phys. Chem. B 2008, 112, 8459-8465. (c) Isare, B.; Linares, M.; Lazzaroni, R.; Bouteiller, L. J. Phys. Chem. B 2009, 113, 3360-3364. (d) Isare, B.; Linares, M.; Zargarian, L.; Fermandjian, S.; Miura, M.; Motohashi, S.; Vanthuyne, N.; Lazzaroni, R.; Bouteiller, L. Chem. Eur. J. 2010, 16, 173-177.

(15) Simic, V.; Bouteiller, L.; Jalabert, M. J. Am. Chem. Soc. 2003, 125, 13148-13154.

(16) (a) Colombani, O.; Bouteiller, L. New J. Chem. 2004, 28, 1373-1382. (b) Isare, B.; Bouteiller, L.; Ducouret, G.; Lequeux, F. Supramol. Chem. 2009, 21, 416-421. (c) Pensec, S.; Nouvel, N.; Guilleman, A.; Creton, C.; Boué, F.; Bouteiller, L. Macromolecules 2010, 43, 2529-2534.

(17) Israelachvili, J. Intermolecular and Surface Forces, Academic Press, $2^{\text {nd }}$ edition, 1992.

(18) The reference temperature was chosen as $\mathrm{T}_{0} * *=27.4^{\circ} \mathrm{C}$, which is the transition temperature of compound Br5 (any other compound can be chosen as reference without affecting the shape of the curve). The transition enthalpy measured for the other compounds is $\Delta \mathrm{h}_{1}\left(\mathrm{~T}_{1}^{* *}\right)$. We assume that $\Delta \mathrm{h}_{1}\left(\mathrm{~T}_{1}^{* *}\right)=\Delta \mathrm{h}_{1}\left(\mathrm{~T}_{0}^{* *}\right)$, which is justified by the fact that there is no heat capacity jump at the tubefilament transition (Figure S3), and therefore that the temperature variation of the enthalpy is negligible..$^{2}$

(19) We could also envisage that the transition is affected by a change of conformation of the alkyl chains.$^{20}$ However, FTIR spectroscopy shows that the proportion of gauche conformations is not affected by the tube to filament transition, whatever the chain length of the bis-urea (see Figure S4 in the Supporting Information). 
(20) Zweep, N.; Hopkinson, A.; Meetsma, A.; Browne, W. R.; Feringa, B. L.; van Esch, J. H. Langmuir 2009, 25, 8802-8809.

(21) To test this assumption, the stability of the monomer to filament transition was studied by ITC (see Figure S5 in the Supporting Information). No influence of the alkyl chain length on the filament stability could be detected. Unfortunately, this test is not conclusive, because the uncertainty of the ITC experiment, although small, is larger than the effect detected by DSC.

(22) Tong, W.; Zheng, Q.; Shao, S.; Lei, Q.; Fang, W. J. Chem. Eng. Data 2010, 55, 3766-3771.

(23) We count as alkyl groups all the methylene and methyl groups coming either from the alkyl or from the alkylene sidechains.

(24) van der Schoot, P.; Bouteiller, L.; Brocorens, P.; Lazzaroni, R. manuscript in preparation. 
For Table of Contents Use Only

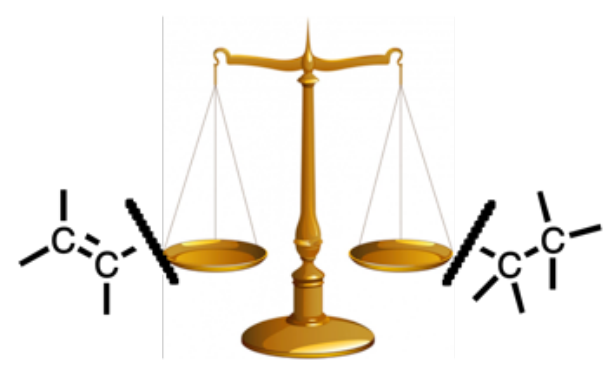

20 